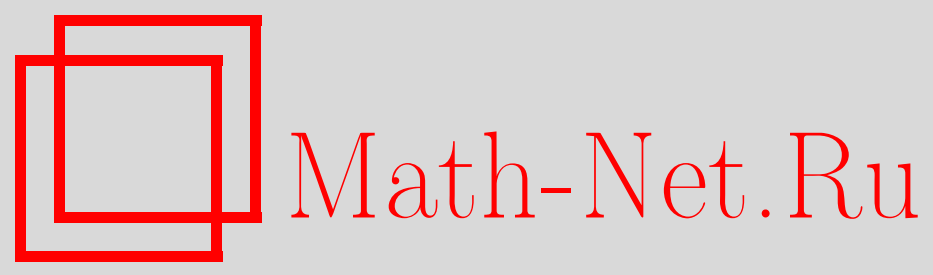

В. В. Федорчук, О брауэровской размерности бикомпактов, Матем. заметки, 2003, том 73, выпуск 2, 295-304

DOI: https://doi.org/10.4213/mzm176

Использование Общероссийского математического портала Math-Net.Ru подразумевает, что вы прочитали и согласны с пользовательским соглашением http://www.mathnet.ru/rus/agreement

Параметры загрузки:

IP: 3.81 .55 .215

26 апреля 2023 г., 18:33:34

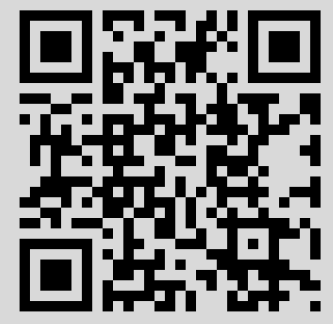




\title{
О БРАУЭРОВСКОЙ РАЗМЕРНОСТИ БИКОМПАКТОВ
}

\author{
В. В. Федорчук
}

\begin{abstract}
Замкнутое множество называется разрезом между двумя непересекающимися множествами, если всякий континуум, пересекающий оба эти множества, пересекает и разрез. Основным результатом статьи является доказательство того, что для любого бикомпакта размерность, определенная индуктивно на основе понятия разреза, не превосходит размерности, определяемой с помощью покрытий.

Библиография: 9 названий.
\end{abstract}

Введение. В 1913 году Брауэр [1] дал первое топологическое определение размерностного инварианта для класса польских (т.е. полных сепарабельных метрических) пространств без изолированных точек или, как говорили тогда, нормальных множеств в смысле Фреше. Он назвал его "Dimensiongrad". Мы будем использовать более краткое обозначение $\mathrm{Dg}$ (все определения см. в п. 1). Брауэр пытался доказать равенство $\operatorname{Dg} I^{n}=n$. Но в его доказательстве неявно предполагалось, что каждьй разрез является перегородкой. После того, как Урысон указал ему на ошибочность этого утверждения, Брауэр [2] заменил в своем определении разрез на перегородку, тем самым исправив свое доказательство $n$-мерности куба $I^{n}$.

Но в классе локально связных польских пространств понятия разреза и перегородки совпадают. Поэтому долгое время считалось, что определение Брауэра эквивалентно урысоновскому определению размерности Ind для класса всех польских локально связных пространств. Об этом было объявлено во введении к книге Гуревича и Волмэна [3]. Затем это утверждение было повторено многими авторами (см. [4]). Недавно выяснилось, что это не верно. А именно, в [4] было доказано, что для всякого $n \geqslant 2$ сушествует локально связное польское пространство $X_{n}$ размерности $\operatorname{Dg} X_{n}=1<n=\operatorname{dim} X_{n}$.

Тем не менее, чуть ранее было показано [5], что брауэровская размерность Dg всетаки совпадает с лебеговой $\operatorname{dim}$ и урысоновской Ind в классе всех метризуемых компактов независимо от их локальной связности (теорема 1.3). Доказательство этого утверждения, состоящее из нескольких строк, является компиляцией четырех фундаментальных фактов теории размерности (сепарабельных) метрических пространств: теоремы счетной суммы замкнутых слагаемых, теоремы о $G_{\delta}$-оболочке той же размерности, аддиционной теоремы и равенства $\operatorname{dim}=$ Ind.

Работа выполнена при поддержке Российского фонда фундаментальных исследований, грант № 00-01-00289. 
В данной заметке доказывается неравенство $\operatorname{dim} X \leqslant \operatorname{Dg} X$ для произвольного бикомпакта $X$ (теорема 3.1). Показывается также (замечание 3.6), что доказать равенство $\operatorname{dim} X=\operatorname{Dg} X$ нельзя даже для бикомпакта $X$ с первой аксиомой счетности.

Все пространства предполагаются тихоновскими, все отображения - непрерьвными. Континуумом назьвается непустой связньй бикомпакт.

1. Предварительные сведения. Пусть даны бикомпакт $X$ и два его непересекающиеся замкнутые подмножества $D$ и $E$. Замкнутоемножество $C \subset X \backslash D \cup E$ назьвается

1) перегородкой в $X$ между $D$ и $E$, если существуют непересекающиеся окрестности $O D$ и $O E$ такие, что $X \backslash C=O D \cup O E$;

2) разрезом в $X$ между $D$ и $E$, если всякий континуум $K$, пересекающийся с $D$ и $E$, пересекается также и с $C$.

Очевидно, что всякая перегородка является разрезом. В то же время простейшие не локально связные плоские континуумы показьвают, что не всякий разрез является перегородкой.

ОПРЕДЕЛЕНИЕ 1.1. Размерность $\operatorname{Dg} X$ бикомпакта $X$ определяется по индукции следуюшим образом:

- $\operatorname{Dg} X=-1$ тогда и только тогда, когда $X=\varnothing$;

- $\operatorname{Dg} X \leqslant n$ тогда и только тогда, когда для любых двух замкнутых непересекающихся множеств $D, E \subset X$ существует разрез $C$ между ними размерности $\operatorname{Dg} C \leqslant n-1$;

- $\operatorname{Dg} X=n$ тогда и только тогда, когда $n$ - наименьшее целое число, для которого $\operatorname{Dg} X \leqslant n$; если не существует целого числа $n$ такого, что $\operatorname{Dg} X \leqslant n$, то $\operatorname{Dg} X=\infty$.

Из того, что всякая перегородка является разрезом, вытекает

ПРЕДЛОЖЕНИЕ 1.2. ДЛя всякого бикомпакта $X$ выполнено неравенство

$$
\operatorname{Dg} X \leqslant \operatorname{Ind} X
$$

ТеОрема 1.3 [5]. Для всякого метризуемого компакта $X$ выполнено равенство

$$
\operatorname{Dg} X=\operatorname{dim} X
$$

Для топологического пространства $X$ через $\operatorname{cov}(X)$ обозначается множество всех его конечных открытых покрытий. Это множество частично упорядочено отношением вписанности. А именно, если $u, v \in \operatorname{cov}(X)$, то $u \leqslant v$ тогда и только тогда, когда $v$ вписано в $u$. Множество $\mathscr{U} \subset \operatorname{cov}(X)$ называется базой множества $\operatorname{cov}(X)$, если $\mathscr{U}$ конфинально в $\operatorname{cov}(X)$. Обратные спектры топологических пространств иногда будем назьвать просто спектрами. Следующее утверждение хорошо известно. Его доказательство можно найти, например, в [6].

ПРЕДЛОЖЕНИЕ 1.4. Пусть бикомпакт $X$ является пределом спектра $S=\left\{X_{a}\right.$, $\left.p_{b}^{a}, A\right\}$, состоящего из бикомпактов и эпиморфизмов. Тогда для всякого $u \in \operatorname{cov}(X)$ существуют $a \in A$ и $v \in \operatorname{cov}\left(X_{a}\right)$ такие, что покрытие $p_{a}^{-1}(v)$ вписано в $и$.

Из предложения 1.4 вытекает 
ПРЕДЛОЖЕНИЕ 1.5. Пусть бикомпакт $X$ является пределом спектра $S=\left\{X_{a}\right.$, $\left.p_{b}^{a}, A\right\}$, состоящего из бикомпактов и эпиморфизмов, и пусть для каждого а $\in A$ зафиксирована база $\mathscr{U}_{a}$ множсества $\operatorname{cov}\left(X_{a}\right)$. Тогда множсество $\cup\left\{p_{a}^{-1}\left(\mathscr{U}_{a}\right)\right.$ : $a \in A\}$ является базой мнохества $\operatorname{cov}(X)$.

Будем говорить, что покрытие $u \in \operatorname{cov}(X)$ nочтu $n$-мерно, если существует покрытие $v \in \operatorname{cov}(X)$ кратности $\leqslant n+1$, вписанное в $u$. Следующее утверждение очевидно.

ПРЕДЛОЖЕНИЕ 1.6. Бикомпакт $X$ имеет размерность $\operatorname{dim} X \leqslant n$ тогда $u$ только тогда, когда в мнохестве $\operatorname{cov}(X)$ имеется база, состоящая из почти п-мерных покрытий.

Напомним одно понятие. Пусть $f: Y \rightarrow Z$-отображение. Малым образом множества $U \subset Y$ относительно отображения $f$ назьвается множество

$$
f^{\#} U \equiv\left\{z \in Z: f^{-1}(z) \subset U\right\}
$$

Если $f(Y)=Z$, то, как легко видеть, $f^{\#} U \subset f(U)$. Если отображение $f$ замкнуто, в частности, если $f$ есть непрерьвное отображение между бикомпактами, то малый образ открытого множества открыт.

ЛЕмма 1.7. Пусть бикомпакт $X$ является пределом обратного спектра $S=$ $\left\{X_{a}, p_{b}^{a}, A\right\}$, состоящего из бикомпактов $и$ әпиморфизмов, $u$ пусть $\operatorname{dim} X \leqslant n$. Тогда для произвольных $a_{0} \in A u и \in \operatorname{cov}\left(X_{a_{0}}\right)$ существует $a_{1} \in A, a_{1} \geqslant a_{0}$, такое, что покрытие $\left(p_{a_{0}}^{a_{1}}\right)^{-1}(u)$ почти п-мерно.

ДокАЗАТЕЛЬСтво. Поскольку $\operatorname{dim} X \leqslant n$, существует конечное открытое покрытие $v$ бикомпакта $X$, вписанное в $p_{a_{0}}^{-1}(u)$ и имеющее кратность $\leqslant n+1$. Согласно предложению 1.4 существуют $a_{1} \in A$ и $w \in \operatorname{cov}\left(X_{a_{1}}\right)$ такие, что покрытие $p_{a_{1}}^{-1}(w)$ вписано в $v$. Можно считать, что $a_{1} \geqslant a_{0}$. Семейство

$$
p_{a_{1}}^{\#} v=\left\{p_{a_{1}}^{\#} V: V \in v\right\}
$$

состоит из открытых множеств и является покрытием пространства $X_{a_{1}}$, поскольку покрытие $p_{a_{1}}^{-1}(w)$ вписано в $v$. Далее, из определения малого образа вытекает, что $p_{a_{1}}^{-1}\left(p_{a_{1}}^{\#} V\right) \subset V$ для всякого $V \in v$. Значит, кратность покрытия $p_{a_{1}}^{\#} v$ не превосходит кратности покрытия $v$. Ясно также, что покрытие $p_{a_{1}}^{\#} v$ вписано в покрытие $\left(p_{a_{0}}^{a_{1}}\right)^{-1}(u)$. Лемма 1.7 доказана.

ОПредЕЛЕниЕ 1.8. Пусть $A$ - направленноевверх частично упорядоченное множество. Говорят, что $A$ сигма-полно, если для каждой возрастающей последовательности $a_{i}, i \in \omega$, его элементов в $A$ существует ее точная верхняя грань. Подмножество $B$ сигма-полного множества $A$ назьвается замкнутым (в $A$ ), если точная верхняя грань произвольной возрастающей последовательности элементов из $B$ принадлежит $B$.

Стандартным примером сигма-полногомножества является множество $\tau^{\omega}$ всех счетных подмножеств несчетного кардинального числа $\tau$, упорядоченное отношением включения. Следующее очевидное утверждение вместе с только что упомянутым примером дает нам практически исчерпьвающий запас сигма-полных множеств. 
ПРЕДЛОЖЕНИЕ 1.9. Замкнутое конфинальное подмножество сигма-полного множества сигма-полно.

ПРЕДЛОЖЕНИЕ 1.10 [7, предложение 1.2]. Пересечение всякого счетного семейства замкнутых конфинальных подмножеств сигма-полного множсества замкнуто и конфинально.

Напомним, что обратный спектр $S=\left\{X_{a}, p_{b}^{a}, A\right\}$ назьвается непрерывным, если для всякого линейно упорядоченного множества $B \subset A$, имеющего точную верхнюю грань $a \in A$, пространство $X_{a}$ естественно гомеоморфно пределу спектра $S \mid B=\left\{X_{b}, p_{c}^{b}, B\right\}$. Расшифруем, что значит "естественно". Обозначим предел спектра $S \mid B$ через $X^{B}$. Предельные проекции $X^{B} \rightarrow X_{b}$ спектра $S \mid B$ обозначим через $p_{b}^{B}$. По определению предела обратного спектра существует единственное отображение $p: X_{a} \rightarrow X^{B}$, удовлетворяющее условию $p_{b}^{B} \circ p=p_{b}^{a}$ для всех $b \in B$. Так вот, это “естественное" отображение $p$ и должно быть гомеоморфизмом.

ОПРЕДЕЛЕНИЕ 1.11 [7]. Непрерьвньй спектр над сигма-полным множеством $A$, состоящий из метризуемых компактов и эпиморфизмов, называется сигма-спектром.

Эффект техники сигма-спектров проявляется только тогда, когда рассматриваются сигма-спектры, пределы которых неметризуемы (в противном случае все сводится к пределам обратных последовательностей). Существующие “в природе" примеры таких сигма-спектров дает следуюшая конструкция, успешно примененная в свое время Щепиньм [7] для исследования различных свойств бикомпактов и отображений между ними.

ПРИмеР 1.12. Пусть $X$ - бикомпакт несчетного веса $\tau$. Вложим его в тихоновский куб $I^{\tau}$. Для счетного множества $a \subset \tau$ обозначим через $X_{a}$ образ бикомпакта $X$ при координатном проектировании $I^{\tau} \rightarrow I^{a}$. Если $b \subset a$, то через $p_{b}^{a}: X_{a} \rightarrow X_{b}$ обозначается ограничение проектирования $I^{a} \rightarrow I^{b}$ на компакт $X_{a}$. Легко видеть, что

$$
S=\left\{X_{a}, p_{b}^{a}, \tau^{\omega}\right\}
$$

есть сигма-спектр, пределом которого является бикомпакт $X$.

Лемма 1.13. Пусть бикомпакт $X$ является пределом сигма-спектра $S=\left\{X_{a}\right.$, $\left.p_{b}^{a}, A\right\}$, и пусть $\operatorname{dim} X \leqslant n$. Тогда для произвольных $a_{0} \in A$ и счетного семейства покрытий $u_{i} \in \operatorname{cov}\left(X_{a_{0}}\right)$ существует $a_{1} \in A, a_{1} \geqslant a_{0}$, такое, что каждое покрытие $\left(p_{a_{0}}^{a_{1}}\right)^{-1}\left(u_{i}\right)$ почти п-мерно.

ДокАЗАТЕльСтво. Согласно лемме 1.7 для всякого $u_{i} \in \operatorname{cov}\left(X_{a_{0}}\right)$ существует $b_{i} \in A$, $b_{i} \geqslant a_{0}$, такое, что покрытие $\left(p_{a_{0}}^{b_{i}}\right)^{-1}\left(u_{i}\right)$ почти $n$-мерно. Поскольку множество $A$ сигма-полно, существует $a_{1} \in A$, мажорируещее все $b_{i}$. Этот элемент $a_{1}$ и будет искомым, поскольку прообраз почти $n$-мерного покрытия почти $n$-мерен. Лемма 1.13 доказана.

ПРЕДЛОЖЕНИЕ 1.14. Пусть бикомпакт $X$ является пределом сигма-спектра $S=\left\{X_{a}, p_{b}^{a}, A\right\}$, и пусть $\operatorname{dim} X \leqslant n$. Тогда множество

$$
A_{n}=\left\{a \in A: \operatorname{dim} X_{a} \leqslant n\right\}
$$

замкнуто и конфинально в $A$. 
ДокАЗАтЕльство. Замкнутость множества $A_{n}$ вытекает из непрерывности спектра $S$ и того, что предел обратного спектра из компактов размерности $\leqslant n$ имеет размерность $\leqslant n$. Предположим, что $A_{n}$ не конфинально $A$. Тогда существует $a_{0} \in A$ такое, что

$$
\operatorname{dim} X_{a} \geqslant n+1 \quad \text { для всех } a \geqslant a_{0} .
$$

Возьмем счетное семейство $u_{i}^{a_{0}}, i \in \omega$, конечных открытых покрытий компакта $X_{a_{0}}$, конфинальное в $\operatorname{cov}\left(X_{a_{0}}\right)$. Согласно лемме 1.13 существует $a_{1} \in A, a_{1} \geqslant a_{0}$, такое, что каждое покрытие $\left(p_{a_{0}}^{a_{1}}\right)^{-1}\left(u_{i}^{a_{0}}\right)$ почти $n$-мерно. То же самое проделаем со счетньг семейством $u_{i}^{a_{1}}$, конфинальным в $\operatorname{cov}\left(X_{a_{1}}\right)$. Найдем $a_{2} \geqslant a_{1}$ такое, что все покрытия $\left(p_{a_{1}}^{a_{2}}\right)^{-1}\left(u_{i}^{a_{1}}\right)$ почти $n$-мерны. Получаем возрастающую последовательность $a_{k}, k \in \omega$. Положим $b=\sup a_{k}$. Тогда семейство

$$
\left\{\left(p_{a_{k}}^{b}\right)^{-1}\left(u_{i}^{a_{k}}\right): i, k \in \omega\right\}
$$

состоит из почти $n$-мерных покрытий, поскольку прообраз почти $n$-мерного покрытия при отображении "на" является почти $n$-мерным покрытием. Из предложения 1.5 и непрерьвности спектра $S$ вытекает, что семейство (1.2) является базой множества $\operatorname{cov}\left(X_{b}\right)$. Поэтому из предложения 1.6 получаем, что $\operatorname{dim} X_{b} \leqslant n$. Но это противоречит условию (1.1). Предложение 1.14 доказано.

ЗАмЕчАниЕ 1.15. Здесь приведено элементарное доказательство предложения 1.14, являющегося основньм утверждением п. 1. Это утверждение можно также доказать, применяя факторизационную теорему Мардешича [8] и спектральную теорему Шепина [7]. Но альтернативное доказательство, использующее два фундаментальных факта общей топологии, не получается короче. А учитьвая то, что весь материал, предваряющий предложение 1.14, существенньп образом используется в дальнейшем, приведенное доказательство наиболее оптимально в контексте данной статьи.

ОПРЕДЕЛЕНИЕ 1.16. Напомним, что для бикомпакта $Y$ через $\exp ^{c} Y$ обозначается множество всех его подконтинуумов, наделенное топологией Вьеториса. Для непустого (метризуемого) $Y$ гиперпространство $\exp ^{c} Y$ является непустым (метризуемым) бикомпактом, содержащим $Y$. Для непрерьвного отображения $f: Y \rightarrow Z$ через $\exp ^{c}(f)$ обозначается непрерьвное (в топологии Вьеториса) отображение $\exp ^{c} Y \rightarrow \exp ^{c} Z$, определяемое равенством $\exp ^{c}(f)(K)=f(K)$. Известно, что конструкция $\exp ^{c}$ является ковариантным функтором в категории Comp бикомпактов и их непрерывных отображений. Более того, этот функтор непрерывен, т.е. перестановочен с операцией перехода к пределу обратного спектра.

ОПРЕДЕЛЕНИЕ 1.17. Напомним, что семейство $\mathscr{B}$ замкнутых подмножеств пространства $X$ назьвается его большой базой, если для всякого замкнутого множества $F \subset X$ и всякой его окрестности $O F$ существует $B \in \mathscr{B}$ такое, что $F \subset B \subset O F$.

ЗАмЕчаниЕ 1.18. Легко видеть, что во всяком бикомпакте $X$ веса $\tau$ сушествует большая база мошности $\tau$.

2. Леммы о разрезающих гранях. Будем пользоваться обозначениями примеpa 1.12. Для $a \in \tau^{\omega}$ положим $\tau_{a}^{\omega}=\left\{b \in \tau^{\omega}: a \subset b\right\}$. В этом пункте множество $a$ фиксировано. Фиксируются также замкнутые непересекающиеся подмножества $D_{a}$ и $E_{a}$ 
компакта $X_{a}$. Полагаем $D=p_{a}^{-1}\left(D_{a}\right), E=p_{a}^{-1}\left(E_{a}\right)$. Фиксируем некоторьй разрез $C$ между $D$ и $E$. Для $a \subset c \subset b \in \tau_{a}^{\omega}$ полагаем

$$
\begin{gathered}
C_{c b}=\left(p_{c}^{b}\right)^{-1} p_{c}(C), \quad D_{b}=p_{b}(D), \quad E_{b}=p_{b}(E), \\
Y_{b}=\left\{K \in \exp ^{c} X_{b}: D_{b} \cap K \neq \varnothing \neq E_{b} \cap K\right\} \\
Z_{b}=\left\{K \in Y_{b}: C_{a b} \cap K=\varnothing\right\} .
\end{gathered}
$$

Лемма 2.1. Для разреза $C$ мехсду $D$ и $E$ существует $a_{0} \in \tau_{a}^{\omega}$ maкое, что $C_{a a_{0}}$ является разрезом межсду $D_{a_{0}}=p_{a_{0}}(D) u E_{a_{0}}=p_{a_{0}}(E)$.

ДокАЗАТЕЛЬСтво. Предположим, что утверждение леммы 2.1 не имеет места. Это означает, что

$$
Z_{b} \neq \varnothing \quad \text { для всякого } b \in \tau_{a}^{\omega} .
$$

Для $a \subset b_{1} \subset b$ положим $e_{b_{1}}^{b}=\exp ^{c}\left(p_{b_{1}}^{b}\right)$. Тогда

$$
Z_{b}=\left(e_{b_{1}}^{b}\right)^{-1} Z_{b_{1}}
$$

В самом деле, в силу (2.1) множества $C_{a b}, D_{b}, E_{b}$ являются прообразами множеств $C_{a b_{1}}, D_{b_{1}}, E_{b_{1}}$ при отображении $p_{b_{1}}^{b}$. Поэтому множество $K \subset X_{b}$ пересекается с одним из множеств $C_{a b}, D_{b}, E_{b}$ тогда и только тогда, когда $p_{b_{1}}^{b}(K)$ пересекается с соответствуюшим из множеств $C_{a b_{1}}, D_{b_{1}}, E_{b_{1}}$. В частности, это так для любого континуума $K \subset X_{b}$. Отсюда равенство $(2.3)$ и вытекает.

Отметим, что множество $Y_{b}$ замкнуто в компакте $\exp ^{c} X_{b}$, а множество $Z_{b}$ открыто в $Y_{b}$. Следовательно, множество $Z_{a}$ является суммой последовательности компактов $F_{k}, k \in \omega$. Положим

$$
B_{k}=\left\{b \in \tau_{a}^{\omega}:\left(e_{a}^{b}\right)^{-1} F_{k} \neq \varnothing\right\}
$$

Из условия (2.2) и равенства (2.3) вытекает

$$
\cup\left\{B_{k}: k \in \omega\right\}=\tau_{a}^{\omega} .
$$

Поскольку $\tau_{a}^{\omega}$, как и никакое сигма-полное множество, нельзя представить в виде счетной суммы не конфинальных подмножеств, из равенства (2.5) вытекает существование $k_{0} \in \omega$ такого, что

$$
B_{k_{0}} \text { конфинально } \tau_{a}^{\omega} \text {. }
$$

Но из (2.3) и (2.4) вытекает, что каждое множество $B_{k}$ насьшено вниз, т.е. $b \in B_{k}$ влечет $b_{1} \in B_{k}$ для всех $b_{1}, a \subset b_{1} \subset b$. Поэтому из (2.6) получаем

$$
B_{k_{0}}=\tau_{a}^{\omega}
$$

Для $b \in \tau_{a}^{\omega}$ положим

$$
\Phi_{b}=\left(e_{a}^{b}\right)^{-1} F_{k_{0}}
$$

Из (2.3), (2.7) и (2.8) вытекает

$$
e_{b_{1}}^{b}\left(\Phi_{b}\right) \subset \Phi_{b_{1}}
$$


(но это не означает, что $\left.e_{b_{1}}^{b}\left(Z_{b}\right) \subset Z_{b_{1}}\right)$.

Положим $\varepsilon_{b_{1}}^{b}=e_{b_{1}}^{b} \mid \Phi_{b}$. Из $(2.9)$ следует, что система $\Sigma=\left\{\Phi_{b}, \varepsilon_{b_{1}}^{b}, \tau_{a}^{\omega}\right\}$ является обратным спектром. Из (2.4) и (2.8) вытекает, что спектр $\Sigma$ состоит из непустых компактов. По теореме Куроша бикомпакт $\Phi=\lim \Sigma$ не пуст. Далее, бикомпакт $\Phi$ в силу непрерьвности функтора $\exp ^{c}$ состоит из континуумов, лежащих в $X$. Возьмем континуум $K \in \Phi$. По определению отображений $e_{b_{1}}^{b}$ и $\varepsilon_{b_{1}}^{b}$ нить спектра $\Sigma$, соответствующая континууму $K$, состоит из континуумов $p_{b}(K), b \in \tau_{a}^{\omega}$. Поэтому из $(2.3)$ вытекает

$$
p_{a}(K) \in F_{k_{0}} \subset Z_{a}
$$

Но это означает, что $p_{a}(K)$ пересекается с $D_{a}$ и $E_{a}$ и не пересекается с $C_{a a}$. Тогда континуум $K$ пересекается с $D=p_{a}^{-1}\left(D_{a}\right)$ и $E=p_{a}^{-1}\left(E_{a}\right)$ и не пересекается с $C \subset p_{a}^{-1}\left(C_{a a}\right)$. Но это противоречит тому, что $C$ является разрезом между $D$ и $E$. Лемма 2.1 доказана.

Лемма 2.2. Существует $b \in \tau_{a}^{\omega}$ такое, что мнохсество $C_{b}=p_{b}(C)$ является разрезом межсду $D_{b} u E_{b}$.

ДокАЗАТЕЛЬСТво. Последовательно применяя лемму 2.1, находим растущую последовательность множеств $a_{k} \in \tau_{a_{k-1}}^{\omega}, k \in \omega$, такую, что

$$
C_{a_{k} a_{k+1}} \text { есть разрез между } D_{a_{k+1}} \text { и } E_{a_{k+1}} \text {. }
$$

Положим $b=\cup\left\{a_{k}: k \in \omega\right\}$. Из (2.1) вытекает, что $p_{a_{k}}^{b}\left(C_{b}\right)=C_{a_{k} a_{k}}$. Следовательно, компакт $C_{b}$ является пределом обратной последовательности $\left\{C_{a_{k}} a_{k}, p_{a_{k-1}}^{a_{k}}, \omega\right\}$. Поэтому

$$
C_{b}=\bigcap\left\{\left(p_{a_{k}}^{b}\right)^{-1} C_{a_{k} a_{k}}: k \in \omega\right\}
$$

Предположим, что $C_{b}$ не является разрезом между $D_{b}$ и $E_{b}$. Тогда существует континуум $K \in Y_{b}$ такой, что $K \cap C_{b}=\varnothing$. Из (2.12) вытекает существование $k \in \omega$ такого, что

$$
K \cap\left(p_{a_{k}}^{b}\right)^{-1} C_{a_{k} a_{k}}=\varnothing
$$

Ho

$$
\left(p_{a_{k}}^{b}\right)^{-1} C_{a_{k} a_{k}}=\left(p_{a_{k+1}}^{b}\right)^{-1}\left(p_{a_{k}}^{a_{k+1}}\right)^{-1} C_{a_{k} a_{k}} .
$$

Поэтому из (2.13) вытекает, что

$$
p_{a_{k+1}}^{b}(K) \cap\left(p_{a_{k}}^{a_{k+1}}\right)^{-1} C_{a_{k} a_{k}}=\varnothing .
$$

Но это противоречит условию $(2.11)$, поскольку $C_{a_{k} a_{k+1}}=\left(p_{a_{k}}^{a_{k+1}}\right)^{-1}\left(C_{a_{k} a_{k}}\right)$ по определению, а $K \in Y_{b}$ влечет $p_{a_{k+1}}^{b}(K) \in Y_{a_{k+1}}$. Лемма 2.2 доказана.

Множество $b$ из леммы 2.2 будем назьвать $(C, D, E)$-разрезающей гранью.

ЛЕмма 2.3. Множество $(C, D, E)$-разрезающих граней замкнуто и конфинальHO $\tau_{a}^{\omega}$. 
ДокАЗАТЕЛьСтво. Конфинальность вытекает из леммы 2.2. Доказательство замкнутости фактически содержится в доказательстве леммы 2.2 , но мы приведем его. Пусть множество $b \in \tau_{a}^{\omega}$ является суммой растущей последовательности $(C, D, E)$-разрезающих граней $b_{k}$. Тогда компакт $X_{b}$ является пределом обратной последовательности $\Sigma=\left\{X_{b_{k}}, p_{b_{k}}^{b_{k+1}}, \omega\right\}$.

Предположив, что $b$ не есть $(C, D, E)$-разрезающая грань, возьмем континуум $K \in Y_{b}$ такой, что $K \cap C_{b}=\varnothing$. Из равенства $X_{b}=\lim \Sigma$ вытекает существование $k$ такого, что $p_{b_{k}}^{b}(K) \cap p_{b_{k}}^{b}\left(C_{b}\right)=\varnothing$. Ясно также, что $p_{b_{k}}^{b}(K) \in Y_{b_{k}}$. Итак, мы нашли континуум $p_{b_{k}}^{b}(K) \subset X_{b_{k}}$, соединяющий множества $D_{b_{k}}$ и $E_{b_{k}}$ и не пересекюшийся с $p_{b_{k}}^{b}\left(C_{b}\right)=C_{b_{k}}$. Но это противоречит тому, что $b_{k}$ является $(C, D, E)$-разрезающей гранью. Лемма 2.3 доказана.

\section{3. Основная теорема.}

ТЕОРемА 3.1. Для всякого бикомпакта $X$ имеет место неравенство

$$
\operatorname{dim} X \leqslant \operatorname{Dg} X
$$

ДокАЗАтЕльСтво. Индукция по $k=\operatorname{dim} X$. Для метризуемого $X$ результат вытекает из теоремы 1.3. Предположим, что неравенство $k \leqslant \operatorname{Dg} X$ доказано для всех $k \leqslant n$. Предположим также, что нашелся неметризуемьй бикомпакт $X$ веса $\tau$, для которого $\operatorname{dim} X=n+1$, но $\operatorname{Dg} X \leqslant n$. В дальнейшем применяем обозначения примера 1.12 . Поскольку $\operatorname{dim} X=n+1$, множество тех $a \in \tau^{\omega}$, что $\operatorname{dim} X_{a} \leqslant n$, не может быть конфинально $\tau^{\omega}$. Следовательно, существует $a \in \tau^{\omega}$ такое, что

$$
\operatorname{dim} X_{b} \geqslant n+1 \quad \text { для всех } b \in \tau_{a}^{\omega} .
$$

Для каждого $b \in \tau_{a}^{\omega}$ возьмем большую счетную базу $\mathscr{B}_{b}$ компакта $X_{b}$ (см. замечание 1.18). Пусть $\left\{\left(D_{b}^{b, i}, E_{b}^{b, i}\right), i \in \omega\right\}$ - семейство всех дизъюнктных пар элементов базы $\mathscr{B}_{b}$. Положим

$$
D^{b, i}=p_{b}^{-1}\left(D_{b}^{b, i}\right), \quad E^{b, i}=p_{b}^{-1}\left(E_{b}^{b, i}\right)
$$

Поскольку $\operatorname{Dg} X \leqslant n$, в $X$ существует разрез $C^{b, i}$ между $D^{b, i}$ и $E^{b, i}$ размерности $\operatorname{Dg} C^{b, i} \leqslant n-1$. Зафиксируем его. По предположению индукции

$$
\operatorname{dim} C^{b, i} \leqslant n-1
$$

Для $b, c \in \tau_{a}^{\omega}$ положим

$$
C_{b}^{c, i}=p_{b}\left(C^{c, i}\right), \quad D_{b}^{c, i}=p_{b}\left(D^{c, i}\right), \quad E_{b}^{c, i}=p_{b}\left(E^{c, i}\right)
$$

Для $c \in \tau_{a}^{\omega}$ положим

$$
\begin{aligned}
& \mathscr{A}_{c, i}=\left\{b \in \tau_{a}^{\omega}: \operatorname{dim} C_{b}^{c, i} \leqslant n-1\right\} \\
& \mathscr{A}^{c, i}=\left\{b \in \tau_{a}^{\omega}: b \text { есть }\left(C^{c, i}, D^{c, i}, E^{c, i}\right) \text {-разрезающая грань }\right\} .
\end{aligned}
$$


Согласно (3.4) бикомпакт $C^{c, i}$ есть предел спектра $\left\{C_{b}^{c, i}, p_{b_{1}}^{b}, \tau_{a}^{\omega}\right\}$. Поэтому из (3.3) и предложения 1.4 вытекает, что $\mathscr{A}_{c, i}$ замкнуто и конфинально $\tau_{a}^{\omega}$. По лемме 2.3 множество $\mathscr{A}^{c, i}$ также замкнуто и конфинально $\tau_{a}^{\omega}$. Поэтому из предложения 1.10 вытекает, что множество

$$
\mathscr{A}_{c}^{i}=\mathscr{A}_{c, i} \cap \mathscr{A}^{c, i}
$$

также замкнуто и конфинально $\tau_{a}^{\omega}$. Положим

$$
\mathscr{A}_{c}=\bigcap\left\{\mathscr{A}_{c}^{i}: i \in \omega\right\} .
$$

Из (3.7) и предложения 1.10 вытекает, что

$$
\mathscr{A}_{c} \text { замкнуто и конфинально } \tau_{a}^{\omega} .
$$

В частности, для любого конечного набора $a_{1}, \ldots, a_{m} \in \tau_{a}^{\omega}$ множество $\mathscr{A}_{a_{1}} \cap \cdots \cap \mathscr{A}_{a_{m}}$ не пусто. Поэтому можно определить последовательность $a_{k} \in \tau_{a}^{\omega}, k \in \omega$, подчиненную условию

$$
a_{k} \in \mathscr{A}_{a_{0}} \cap \cdots \cap \mathscr{A}_{a_{k-1}} .
$$

Из определения множеств $\mathscr{A}_{c}$ вытекает свойство

$$
a_{k-1} \subset a_{k}
$$

Положим $b_{0}=\bigcup\left\{a_{k}: k \in \omega\right\}$ и покажем, что

$$
\operatorname{dim} X_{b_{0}} \leqslant n
$$

Для этого в силу теоремы 1.3 достаточно доказать следующее

УТВЕРЖДЕНИЕ (*). Для любых двух непересекающихся замкнутых подмножеств $D$ и $E$ компакта $X_{b_{0}}$ межсду ними существует разрез $C$ размерности $\operatorname{dim} C \leqslant n-1$.

ДокАЗАтЕЛьСтво. Поскольку $X_{b_{0}}=\lim \left\{X_{a_{k}}: k \in \omega\right\}$, существует $k \geqslant 1$ такое, что $p_{a_{k}}^{b_{0}}(D) \cap p_{a_{k}}^{b}(E)=\varnothing$. Но $\mathscr{B}_{a_{k}}$ является большой базой компакта $X_{a_{k}}$. Поэтому существует дизъюнктная пара $D_{a_{k}}^{a_{k, i}}, E_{a_{k}}^{a_{k, i}} \in \mathscr{B}_{a_{k}}$ такая, что $p_{a_{k}}^{b_{0}}(D) \subset D_{a_{k}}^{a_{k, i}}$, $p_{a_{k}}^{b_{0}}(E) \subset E_{a_{k}}^{a_{k, i}}$.

Покажем, что $C_{b_{0}}^{a_{k}, i}$ и есть искомый разрез $C$. Согласно (3.9) для любого $m>k$ имеем $a_{m} \in \mathscr{A}_{a_{k}} \subset \mathscr{A}^{a_{k}, i}$.

Поэтому из (3.6) вытекает, что $a_{m}$ есть $\left(C^{a_{k}, i}, D^{a_{k}, i}, E^{a_{k}, i}\right)$-разрезающая грань. Из (3.10), определения множества $b_{0}$ и леммы 2.3 вытекает, что $b_{0}$ также является $\left(C^{a_{k}, i}\right.$, $\left.D^{a_{k}, i}, E^{a_{k}, i}\right)$-разрезающей гранью, т.е. $C_{b_{0}}^{a_{k}, i}$ есть разрез между $D_{b_{0}}^{a_{k}, i}$ и $E_{b_{0}}^{a_{k}, i}$. Тогда $C_{b_{0}}^{a_{k}, i}$ является разрезом и между меньшими множествами $D$ и $E$. Из того же условия (3.9) вытекает, что $a_{m} \in \mathscr{A}_{a_{k}, i}$ при $m>k$.

Следовательно, $\operatorname{dim} C_{a_{m}}^{a_{k}, i} \leqslant n-1$ согласно определению множества $\mathscr{A}_{c, i}$ (cм. (3.5)). Но $C_{b_{0}}^{a_{k}, i}=\lim \left\{C_{a_{m}}^{a_{k}, i}: m>k\right\}$. Поэтому $\operatorname{dim} C_{b_{0}}^{a_{k}, i} \leqslant n-1$. Утверждение $(*)$ доказано.

Значит, имеет место неравенство (3.11). Но это противоречит неравенству (3.1). Теорема 3.1 доказана.

Из теоремы счетной суммы для размерности dim вытекает 
СлЕДСТВИЕ 3.2. Для всякого б-бикомпактного пространства $X$ выполнено неравенство

$$
\operatorname{dim} X \leqslant \operatorname{Dg} X
$$

СЛЕДСТВИЕ 3.3. Для всякого локально бикомпактного линделёфова пространства $X$ выполнено неравенство

$$
\operatorname{dim} X \leqslant \operatorname{Dg} X
$$

Из следствия 3.3 и очевидной теоремы дискретной суммы для брауэровской размерности вытекает

СЛЕДСТВИЕ 3.4. Для всякого локально бикомпактного паракомпактного пространства $X$ выполнено неравенство

$$
\operatorname{dim} X \leqslant \operatorname{Dg} X
$$

СлЕДСТВИЕ 3.5. Если $X-$ бикомпакт $u \operatorname{Dg} X=1, \operatorname{mo} \operatorname{dim} X=1$.

Действительно, это утверждение вытекает из теоремы 3.1 и того, что равенства $\operatorname{Dg} X=0$ и $\operatorname{dim} X=0$ равносильны.

ЗАмечАниЕ 3.6. Существуют бикомпакты $X$, для которых равенство $\operatorname{Dg} X=\operatorname{dim} X$ не имеет места. В самом деле, в качестве $X$ можно взять двумерньй бикомпакт $B_{2}$ без промежуточных размерностей [9]. Тогда $X$ не содержит замкнутых одномерных подмножеств. Отсюда, в силу индуктивного определения инварианта Dg вытекает, что $\operatorname{Dg} X=\infty$, в то время, как $\operatorname{dim} X=2$. Отметим также, что бикомпакт $B_{2}$ строится без дополнительных теоретико-множественных предположений и удовлетворяет первой аксиоме счетности.

\section{СПИСОК ЦИТИРОВАННОЙ ЛИТЕРАТУРЫ}

[1] Brouwer L.E. J. Über den natürlichen Dimensionsbegriff // J. Reine Angew. Math. 1913. V. 142. P. $146-152$.

[2] Brouwer L. E. J. Berichtung zur Abhandlung "Über den natürlichen Dimensionbegriff", dieses Journal Bd. 142 (1913), S. 146-152 // J. Reine Angew. Math. 1924. V. 153. P. 253.

[3] Гуревич В., Волмэн Г. Теория размерности. М.: ИЛ, 1948.

[4] Fedorchuk V. V., van Mill J. Dimensiongrad for locally connected Polish spaces // Fund. Math. 2000. V. 163. № 1. P. 77-82.

[5] Федорчук В. В., Левин М., Шепин Е. В. О брауэровском определении размерности // УМН. 1999. Т. 54. № 2. С. 193-194.

[6] Александров П. С., Пасынков Б. А. Введение в теорию размерности. М.: Наука, 1973.

[7] Шепин Е. В. Функторы и несчетные степени компактов // УМН. 1981. Т. 36. № 3. С. 3-62.

[8] Mardesic S. On covering dimension and inverse limits of compact spaces // Illinois J. Math. 1960. V. 4. № 2. P. 278-291.

[9] Федорчук В. В. Бикомпакты без промежуточных размерностей // Докл. АН СССР. 1973. Т. 213. № 4. С. $795-797$. 